\title{
How Well Do Oscillator Models Capture the Behaviour of Biological Neurons?
}

\author{
David Bhowmik \\ Department of Computing, Imperial College London, \\ 180 Queen's Gate, London SW7 2AZ, \\ United Kingdom \\ d.bhowmik10@imperial.ac.uk
}

\author{
Murray Shanahan \\ Department of Computing, Imperial College London, \\ 180 Queen's Gate, London SW7 2AZ, \\ United Kingdom \\ m.shanahan@imperial.ac.uk
}

\begin{abstract}
It has been proposed that groups of neurons firing synchronously provide a mechanism that underlies many cognitive functions such as attention, associative learning, and working memory, as well as opening up communication channels between neuron groups. A mathematical abstraction that is gaining increasing acceptance for modeling neural information processing is the Kuramoto oscillator model, which can be used as an elementary unit to represent populations of oscillatory neurons. Whilst the Kuramoto model is widely used to capture fundamental properties of the collective dynamics of interacting communities of oscillatory neurons, the question arises as to how well it performs this role. This paper aims to address that question experimentally by using neural models to replicate the most fundamental of Kuramoto's findings, in which he showed that for any number of oscillators there is a critical coupling value $K_{c}$ below which the oscillators are fully unsynchronized and another critical coupling value $K_{L} \geq K_{c}$ above which all oscillators become fully synchronized. In this study, we replace Kuramoto oscillators with oscillating populations both of quadratic integrate-and-fire neurons and of Hodgkin-Huxley neurons to establish whether Kuramoto's findings still hold in a more biologically realistic setup. The individual oscillators use a pyramidal inter-neuronal gamma architecture designed using a novel evolutionary technique.
\end{abstract}

Keywords-synchronization; complexity; Kuramoto oscillators; spiking neurons.

\section{INTRODUCTION}

Studies of spiking activity suggest that local field potentials (LFP) arise from the combined activity of large numbers of neurons distributed over a region of the cortex. Recent studies have pointed to the potentially strong correlation between the blood-oxygen-level dependence signal (BOLD) and gamma LFP. [1]. Functional magnetic resonance imaging (fMRI) based upon BOLD has shown significant correlations between distinct anatomical regions [2]. It has been proposed that such disparate groups of neurons firing synchronously together provide a mechanism that underlies many cognitive functions such as attention [3], associative learning [4], working memory [5], and the formation of episodic memory [6, 7]. Recently, a role for synchronization has been proposed in opening up communication channels between neuron groups [8]. Further to this, it has been suggested that transient periods of synchronization and desynchronization provide mechanism for dynamically forming coalitions of functionally related neural areas [9].

An increasingly common level of abstraction for modeling neural information processing is one in which phase oscillators are used as elementary units representing populations of oscillatory neurons $[10,11]$. A popular phase oscillator model used to capture the collective dynamics of such interacting communities is the Kuramoto model [10, 12, 13]. For example, Cabral et al [14] use a Kuramoto model simulation to explore the relationship between the slow modulation of gamma-band activity in the functional connectivity of a resting state network as seen in fMRI results and its relationship to anatomical connectivity. Investigating metastable chimera states in smallworld topologies akin to those that appear in the brain, Shanahan [9] built a community-structured network of Kuramoto oscillators and identified, as did Cabral et al [14], the rich dynamics that results from the interplay between longrange connectivity of a large-scale network and interactions at a local level. In order to engage neuroscience with insights into the situated and embodied nature of cognitive dynamics Santos et al [15] explored metastable dynamical regimes in a network of Kuramoto oscillators that was embodied in an agents sensorimotor loop.

Although the Kuramoto model is widely used to capture fundamental properties of the collective dynamics of interacting communities of oscillatory neurons, the question arises as to how well or accurately it performs this role. This paper aims to address that question by using neural models to replicate the most fundamental of Kuramoto's findings. In a much cited monograph, Kuramoto [12] showed that for an infinite number of oscillators with different intrinsic frequencies that are all uniformly connected with one another, there is a critical coupling value $\mathrm{K}_{\mathrm{c}}$ below which the oscillators are fully unsynchronized. Further to this there is another critical coupling value $\mathrm{K}_{\mathrm{L}} \geq \mathrm{K}_{\mathrm{c}}$ above which all oscillators become fully synchronized. [16] In this paper we emulate this result, but using populations of oscillating neurons in place of the simpler Kuramoto oscillators. The classic experiment is performed first using quadratic integrate-and-fire (QIF) neurons [17] and then using the Hodgkin-Huxley [18] neuron model (HH). The two models capture the properties of Type I and Type II neurons respectively. 


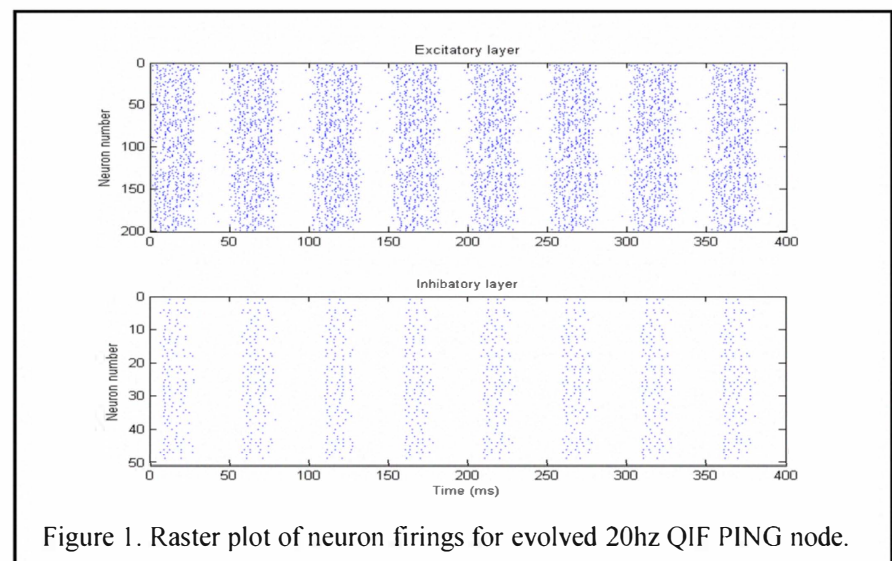

The paper is organized as follows: In section II we describe both the pyramidal inter-neuronal gamma (PING) architecture used to generate oscillations in an individual neural cluster and the evolutionary technique we have used to generate a collection of oscillatory nodes of different frequencies to be drawn from and used in the final experiment. In section III we describe the set up for the final Kuramoto experiments. Following this section IV details the results. Section V presents a discussion, and section VI describes the materials and methods used in our experiments.

\section{ARCHITECTURE OF NEURAL OSCILLATORY NODES}

\section{A. Pyramidal inter-neuronal gamma}

Although studies have shown that groups of neurons firing together rhythmically can occur because of intrinsic firing patterns of excitatory principal cells or common input from a pacemaker, it is more common both in the cortex and the hippocampus that rhythmic firing happens as an emergent property of interactions between excitatory principal cells and inhibitory interneurons. Variations of this mechanism, known as pyramidal inter-neuronal gamma (PING), can give rise to both faster gamma (30-100 hz) and slower theta (4-8 hz) oscillations in the cortex and the hippocampus [7].

Excitatory neurons drive the entire local network, including inhibitory interneurons. The most strongly driven inhibitory neurons will fire first and provide inhibition to numerous other inhibitory neurons. The inhibitory effect on all these neurons will disappear at approximately the same time. Affected inhibitory neurons will then fire roughly together, causing large numbers of inhibitory neurons to be entrained to a rhythm within just a few oscillatory cycles [19]. This rhythmically synchronized inhibition also affects the network's excitatory neurons with a fast and strong synaptic input [20] thus leaving only a short window for the excitatory neurons to fire after one period of inhibition wears off and before the next one starts [21].

Gamma-aminobutyric acid (GABA) is one of the two neurotransmitters that facilitate inhibition in mammal brains. The synaptic reversal potential of GABA receptor currents varies widely among cell types, and probably even between compartments within same cell. When the synaptic reversal potential is below the resting potential, inhibition will be hyperpolarizing. GABAergic synapses can be excitatory if the

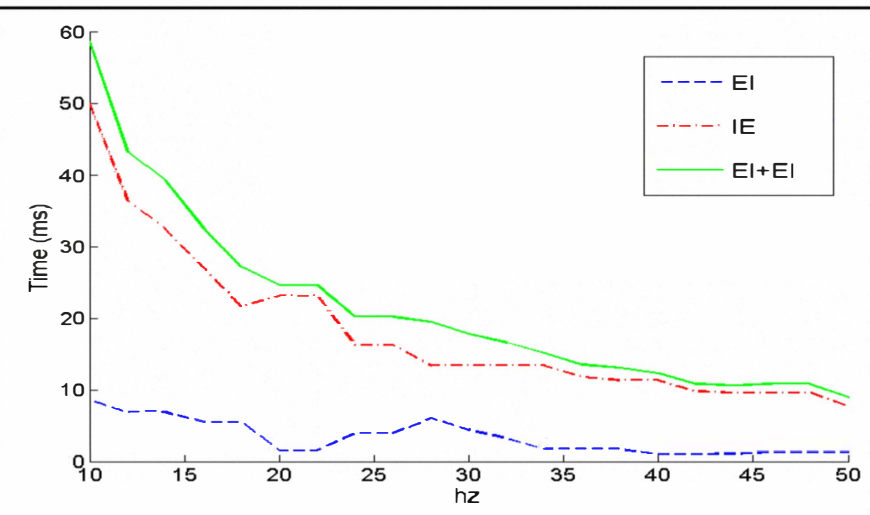

Figure 2. Mean evolved delays for QIF PING nodes.

synaptic reversal potential is above the action potential threshold. If the synaptic reversal potential lies between the resting potential and the action potential threshold, GABAergic synapses will be shunting $[22 ; 23]$. For the models used in this paper, all synapses connecting from inhibitory neurons effectively use a synaptic reversal potential that is below the resting state $(-65 \mathrm{mV})$ of the target neuron, and as such inhibition is hyperpolarising (see section VIB for model details).

\section{B. Evolutionary generation of oscillatory popualtions}

Whilst the general PING architecture is well understood, the specific details required for both particular oscillatory frequencies and neuron model varies and involves a large space of parameter values within the general PING framework. In order to provide a wide range of different intrinsic oscillatory frequencies for the nodes used in the critical coupling experiment, it was decided to evolve within biologically plausible bounds every alternate oscillatory frequency between $10 \mathrm{hz}$ and $50 \mathrm{hz}$ for both QIF and HH models. This was done whilst making each neural network evolved comply to the general PING architecture mentioned above. All neural populations used an excitatory layer of 200 neurons and an inhibitory layer of 50 neurons. The excitatory layer drives the entire network and so is the only one to receive external input. The input is generated from a poison process with parameter $\lambda$ $=3.5 / 0.8$. For QIF models the inputs are scaled by 8 and for the HH models the inputs are scaled by 15 in order to provide sufficient stimulus to induce firing. The networks were wired up with connections between inhibitory neurons (II), from excitatory to inhibitory neurons (EI) and from inhibitory to excitatory neurons (IE). In addition to the synaptic weight, a scaling factor of 5 was used on all synaptic current in the oscillatory populations for both QIF and $\mathrm{HH}$ models to simulate networks of a larger size than we could feasibly simulate otherwise.

The parameters that were evolved were the synaptic weights and delays. Both of these were generated during genome expression of each individual in each generation using a normal distribution, with the means and variances for the weights and the delays being the parameters in the genome evolved. Weights were bound to evolve values between 0 and 1 for excitatory connections and 0 and -1 for inhibitory connections. Long delays are quite unrealistic for a cluster of 


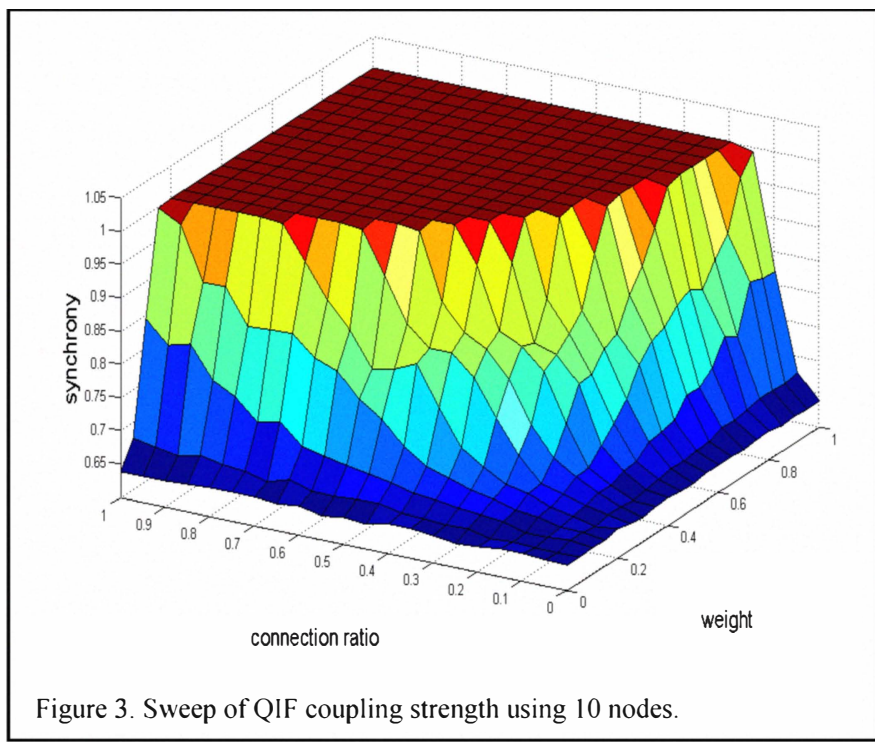

neurons in which all neurons are anatomically close together. In the cortex synaptic latency ranges from $0.2 \mathrm{~ms}$ to $6 \mathrm{~ms}$ [24]. In order to produce realistic results, excitatory delays were bounded between $1 \mathrm{~ms}$ and $10 \mathrm{~ms}$. The IE and II delays were allowed to have a maximum value of $50 \mathrm{~ms}$ to simulate the effect of slow inhibitory interneurons, the behavior of which was otherwise not modeled.

The fitness function for the genetic algorithm consisted first of taking the spike firing times of the excitatory population and converting it to a continuous time-varying signal. This was achieved by binning the spikes over time, and then passing a Gaussian smoothing filter over the binned data. Next a Fourier transform was performed on the signal to produce the frequency spectrum of the signal. The main fitness term was calculated by creating a scaled Gaussian centered around the desired frequency $f$ in the spectrum of the form:

$$
c l i p=20 \mathrm{G}\left(f, \frac{1}{1000}\right)
$$

The frequency spectrum $s$ was subtracted from this and normalized:

$$
\text { fitness }=\frac{-a b s(\text { clip }-s)}{\sum \text { clip }}
$$

An extra penalty term was introduced to discourage frequencies outside the desired range. This was achieved by multiplying the frequency spectrum by -0.002 in the areas further away from the desired frequency whilst ignoring the area at and immediately around the desired frequency. The result was then normalized and added to the main fitness term.

The evolutionary population consisted of 20 individual genomes. For each generation, each individual was tested for $2000 \mathrm{~ms}$ of simulated time. After this each individual was rated for fitness and probabilistically selected for the next generations parents based upon their fitness ranking. Crossover was performed on parent genomes after which mutation was applied to the offspring with a probability of 0.1 .

An example of the spiking behavior of evolved population oscillating at $20 \mathrm{hz}$ can be seen in figure 1 . All evolved weight for QIF solutions had very high means and small variances, whereas the $\mathrm{HH}$ solution showed greater variation in the weight means across evolved solutions for different frequencies, indicating greater sensitivity in the model and solution in that they require a very specific balance of the parameters for each particular solution. The means for the delays evolved for both QIF and HH solutions had a similar form, from which can be concluded that the EI mean delay + IE mean delay $\approx 1000 / 2 f$ with deviations being accounted for by delay variances as well as the fact that some evolved individuals did not evolve to peak fitness. The delay mean results for QIF neurons are shown in figure 2 .

\section{EXPERIMENTAL SETUP}

\section{A. Overview}

Our experiment attempted to reproduce Kuramoto's findings [12] in which he showed that, for an infinite number of oscillators with different intrinsic frequencies and phases that are all uniformally connected with one another using the same coupling strength, a) there is a critical coupling value $\mathrm{K}_{\mathrm{c}}$ below which the oscillators are fully unsynchronized, and $b$ ) there is another critical coupling value $\mathrm{K}_{\mathrm{L}} \geq \mathrm{K}_{\mathrm{c}}$ above which all oscillators become fully synchronized [16].

\section{B. Assessing the notion of neural coupling strength}

Replicating this work experimentally with neural systems we are faced with one immediate question. How does the notion of coupling strength in Kuramoto's oscillator model, in which each node is a simple phase oscillator, relate to a neural model in which each oscillatory node is made up of many neurons? We have two options: the coupling between two nodes may refer to the number of synaptic connections between the neurons, or it may refer to the strength of these synaptic connections.

To address this issue we first present results from a parameter sweep of connection ratios and synaptic weights. At each sweep point the experiment takes 10 QIF oscillator nodes that have each been previously evolved to oscillate at a different frequency using a PING architecture as described in section IIB above. Each node is chosen by frequency from a uniform distribution ranging between $10 \mathrm{hz}$ and 50hz. The excitatory layers in each node are connected to one another with a given connection ratio to form a network of nodes. A connection ratio of 1 defines an all to all connectivity between two nodes so that each neuron in one node's excitatory layer is connected to each neuron in another node's excitatory layer. Once the connections have been established they are given a weight as defined by the point in the sweep. The sweep is two dimensional, ranging from a connection ratio of 0 to 1 and a synaptic weight from 0 to 1 . An increment of 0.05 is used for both the connection ratio and the synaptic weight strength in 


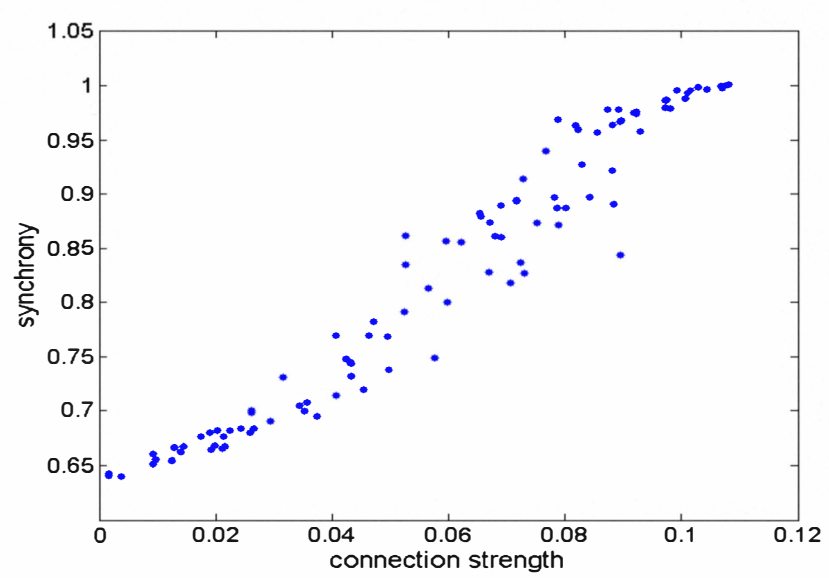

Figure 4. Synchrony of network for a sweep of weights for 64 QIF nodes selected from a Gaussian distribution.

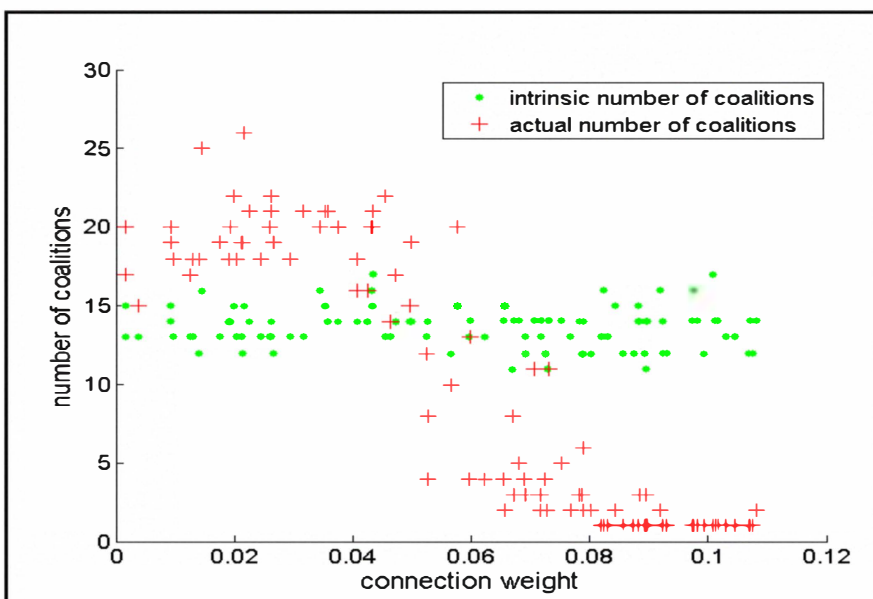

Figure 6. Unique frequency coalitions for QIF networks.

the sweep. At each point in the sweep the overall synchrony of the network is measured as described in the materials and methods detailed in section VI.

The result of the sweep is shown in figure 3 . It can be seen that connection ratio and weight have a similar effect with neither showing a marked importance over the other and with the graph appearing symmetrical. From this result it is safe to assume that our use of both connection ratio and synaptic weight strength in the main Kuramoto experiment will not be biased by using a prescribed value for the connection ratio and sweeping across only the synaptic weight strength in order to explore Kuramoto notion of coupling strength. As such all further experiments will use a connection ration of 0.2 and simply sweep the synaptic weight parameter.

\section{Critical $K$ experimental setup}

Kuramoto calculated analytically the critical values in the case of an infinite number of oscillators connected all-to-all for a few well-known distributions of intrinsic oscillator frequencies [16]. For simplicity, Kuramoto assumed that the distribution of oscillator intrinsic frequencies was unimodal and symmetric about its mean frequency, as in a Gaussian

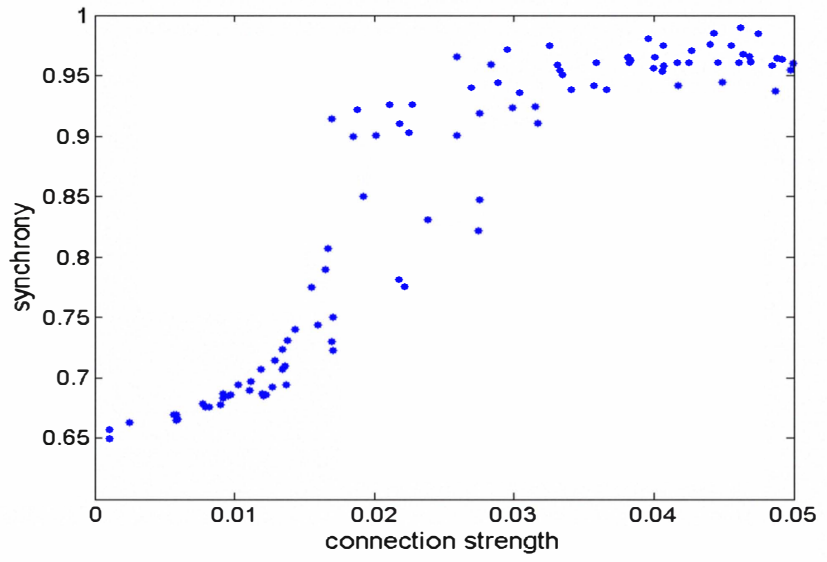

Figure 5. Synchrony of network for a sweep of weights for $64 \mathrm{HH}$ nodes selected from a Gaussian distribution.

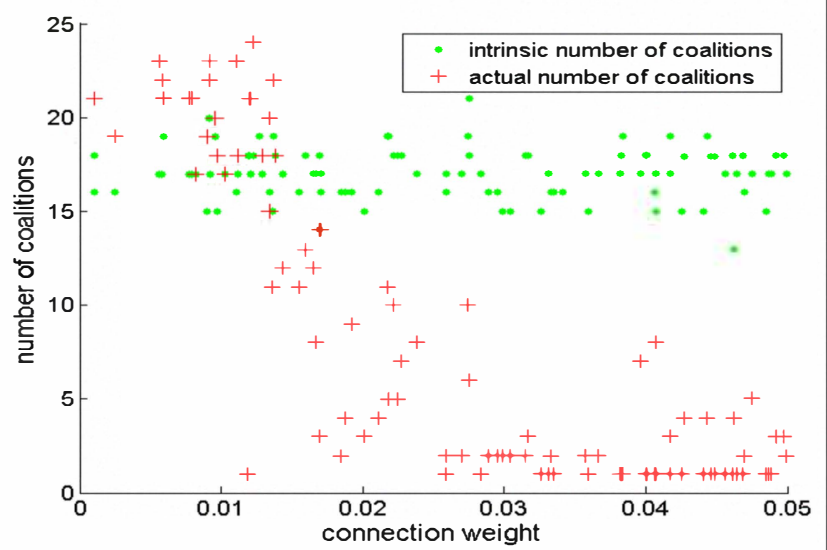

Figure 7. Unique frequency coalitions for $\mathrm{HH}$ networks.

distribution for example [13]. We have evolved PING architectures for every alternate frequency between $10 \mathrm{hz}$ and $50 \mathrm{hz}$ for both QIF and $\mathrm{HH}$ neuron models. In line with Kuramoto's specification we selected from these oscillators using a Gaussian distribution with a mean of $30 \mathrm{hz}$. The variance we chose in order to ensure a good spread of different oscillator frequencies was $10 \mathrm{hz}$.

In all our experiments we used 64 neural oscillator nodes to form a network of nodes. Each node receives external input to its excitatory layer as with the evolutionary setup described in section IIB. The external input along with the PING architectures induces the intrinsic oscillation at the frequency the node was evolved for. The phase of each oscillator was determined by the time at which external input to the oscillator was started, which varied from $0 \mathrm{~ms}$ to $100 \mathrm{~ms}$. The slowest oscillator was $10 \mathrm{hz}$ and therefore a random start point ranging from $0 \mathrm{~ms}$ to $100 \mathrm{~ms}$ allowed for $10 \mathrm{hz}$ oscillators (as well as all oscillators of higher frequency) to be completely out of phase with each other. The neurons in the excitatory layers of each node were synaptically connected to the neurons in the excitatory layers of each other node with a connection ratio of 0.2. The experiments involved a sweep of synaptic weights for 

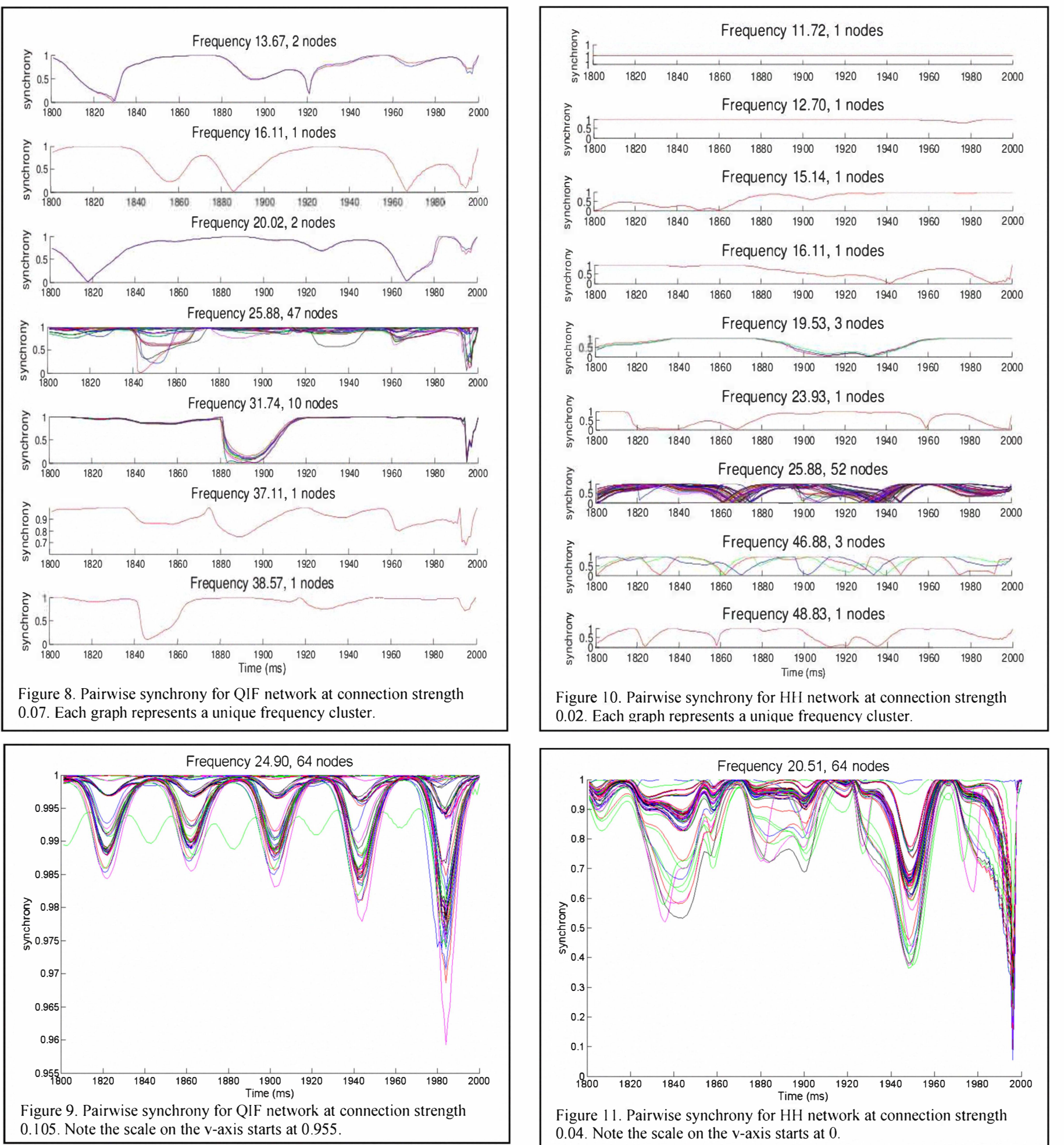

all inter-node connections. These weights were all set to the same value for each iteration in the experimental sweep with each iteration changing this synaptic weight value. At each point in the sweep the overall synchrony of the network was measured as described in the materials and methods in section VI. The network was simulated for $2000 \mathrm{~ms}$ for each iteration of the sweep. Each network comprised 16000 neurons and $36,256,000$ synapses.

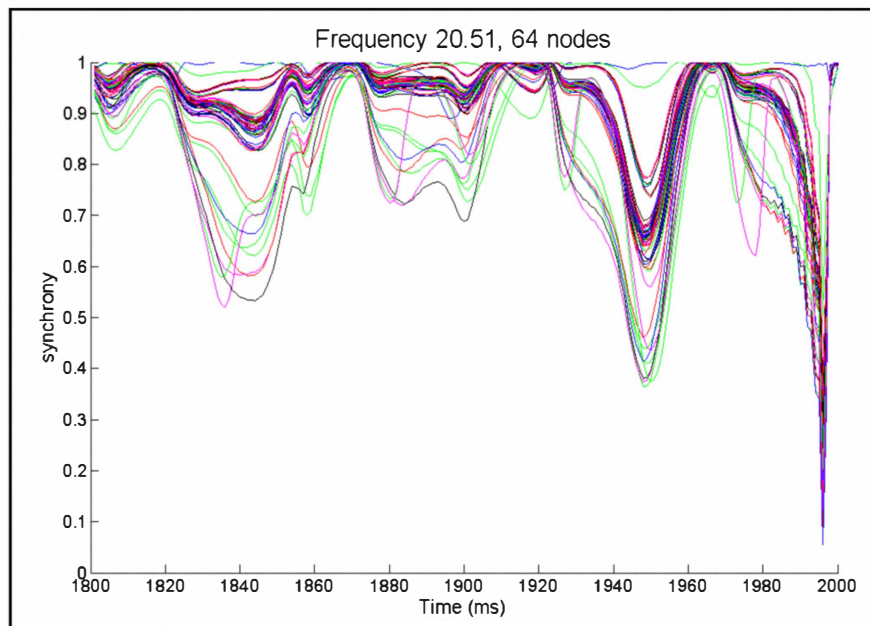

Figure 11. Pairwise synchrony for $\mathrm{HH}$ network at connection strength 0.04 . Note the scale on the $v$-axis starts at 0 .

\section{RESULTS}

Weight sweeps were performed for both QIF and $\mathrm{HH}$ networks with 100 sample weights for each model type. After an iteration in the sweep of a particular high coupling value, both network models exhibited "saturation", meaning that all excitatory neurons in all nodes were firing continuously. The results shown here display data up to the respective point of 


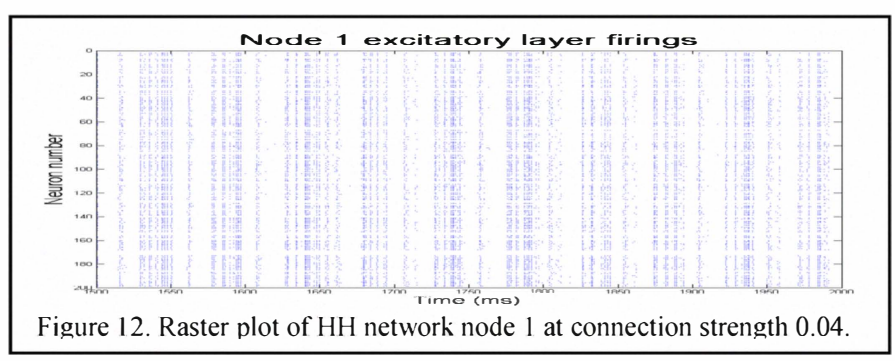

saturation for each model type as data beyond this point is not noteworthy.

Both network types have an initial synchrony value of 0.65 which corresponds to no synchrony apart from coincidental phase alignment. The weight sweeps for the QIF model can be seen in figure 4 and the weight sweeps for the $\mathrm{HH}$ model can be seen in figure 5 . Both models show a steady increase in synchrony until saturation is reached, which is at weight 0.19 for QIF networks and 0.45 for $\mathrm{HH}$ networks. The data is best understood by comparing the number of coalitions, where within each coalition the nodes are oscillating at the same unique frequency. As we are using discrete intrinsic oscillatory frequencies selected from a Gaussian distribution, in one run for one weight setting there may be several nodes with the same intrinsic frequency. Therefore comparing the number coalitions of oscillators sharing the same frequency during the experiment is only meaningful in relation to the number of coalitions that can be formed from the intrinsic frequencies on each trial. Figure 6 shows the number of frequency coalitions both intrinsic and actual results from the experiment during the sweep for QIF networks. Figure 7 presents the same data for $\mathrm{HH}$ networks. Weight 0.05 for QIF networks and weight 0.015 for $\mathrm{HH}$ networks marks the critical point $\mathrm{K}_{\mathrm{c}}$ beyond which the number of coalitions becomes less than the number of intrinsic frequency coalition. This indicates that nodes are affecting one another so as to deter each other from their natural frequency towards a shared frequency. It is interesting to note that prior to the point $\mathrm{K}_{\mathrm{c}}$ both network models display the ability to pull apart from their intrinsic frequency groups into more frequencies than prescribed by the initial Gaussian selection. The QIF model reaches a point of full synchrony $\mathrm{K}_{\mathrm{L}}$, at 0.095 , beyond which one frequency exist for all nodes and is maintained until saturation at 0.19 . The $\mathrm{HH}$ model displays full synchrony at $\approx 0.026$ showing only one frequency group. It is clear from figures 6 and 7 that $\mathrm{K}_{\mathrm{L}}$ for the $\mathrm{HH}$ model is much less well defined than for the QIF model.

Taking a closer look, we present plots of pairwise synchrony between node 1 in the network and all other nodes at particular weight points in the sweep. Figure 8 shows pairwise synchrony for the QIF network at weight 0.07 , a point after $\mathrm{K}_{\mathrm{c}}$. Each sub-plot shows the nodes in a coalition sharing the same frequency. As can be seen the behavior is very tight with the nodes in each group moving closely together both in the same pattern and with little offset indicating matching phases as well. However they do not maintain a constant offset from the phase of node 1 indicating that whilst the frequency and phase of a coalition remains the same within itself, a more complex synchronized behavior is at play in the network of nodes as a whole. A few desynchronous moments appear for the group with frequency $25.88 \mathrm{hz}$ indicating that the nodes themselves are not fully stable at this coupling strength. Figure 9 shows the pairwise synchrony for the QIF network at weight 0.105 . As can be seen, all nodes are synchronous with minor periodic deviations of up to 0.05 from maximum synchrony resulting in general synchrony over 0.955 which is very high. The HH model behaves a lot more noisily. Figure 10 displays the pairwise synchrony at weight 0.02 , a point beyond $\mathrm{K}_{\mathrm{c}}$. Nodes sharing frequency $25.88 \mathrm{hz}$ show distinct phase offset, whereas nodes sharing frequency $46.88 \mathrm{hz}$ do not appear very synchronous at all. A closer inspection of the spiking behavior for this group shows that whilst they share a common main frequency their firing patterns are much noisier with other less well defined frequencies present. Similar behavior is seen in figure 11 which shows the HH network at weight 0.04 , a point at which all nodes share the same frequency. Again on inspection of the firing behavior shown in the raster plot in figure 12, a much noisier behavior is seen in which other frequencies underlie the main frequency of $20.51 \mathrm{hz}$.

\section{DISCUSSION}

The experimental results presented in this paper show that suitably connected oscillatory populations of Type I and Type II neurons broadly conform to critical coupling findings found in Kuramoto oscillators. However both Type I and Type II models display much greater spectral complexity than that which the simple Kuramoto oscillator can capture. In fact, the more detailed and biologically plausible the model is then the greater the spectral complexity. This greater complexity may have implications for neural simulation using the Kuramoto abstraction, as this simple model only partially captures the range of temporal phenomena we find with biologically plausible spiking models.

\section{MATERIALS AND METHODS}

\section{A. Neural models}

Hansel et al [25] distinguish two types of neuron responses. The first type of neuron (Type I) always responds to small depolarization by advancing the next spike. An example of such a neuron is the integrate-and-fire model. The second type (Type II) is exemplified by the Hodgkin-Huxley model in which there is a negative region just after the refractory period, where a depolarization delays the firing of the next spike because the delayed rectifier potassium current is greater than the sodium current, while an excitatory post-synaptic potential received at a later time advances the firing. In this paper both Type I and Type II models are assessed.

\section{1) Quadratic integrate-and-fire neurons}

The QIF model [17] displays Type I neuron dynamics [26]. The time evolution of the neuron membrane potential is given by:

$$
\frac{d V}{d t}=\frac{1}{\tau}\left(V-V_{r}\right)\left(V-V_{t}\right)+\frac{I}{C}
$$


where $V$ is the membrane potential, with $V r$ and $V t$ being the resting and threshold values respectively. $C$ is the capacitance of the cell membrane. $\tau$ is the membrane time constant such that $\tau=R C$ with $R$ being the resistance. $I$ represents a depolarizing input current to the neuron.

An action potential occurs when $V$ reaches a value $V_{\text {peak }}$ at which point it is reset to value $V_{\text {reset. The }}$ QIF model is equivalent to the theta neuron model described by Ermentrout and Kopell [27] if one sets the reset condition $V_{\text {peak }}=\infty$ and $V_{\text {reset }}=-\infty$. Like Börgers and Kopell [28] we use values $V_{r}=V_{\text {reset }}=0$ and $V_{t}=V_{\text {peak }}=1$, which reduces equation (1) to:

$$
\frac{d V}{d t}=a V(V-1)+\frac{I}{C}
$$

Here $a=\frac{1}{\tau}$ and is set to the value 2 for all experiments carried out in the paper. When working with the QIF model we assume a membrane potential between $V_{r}=-65 \mathrm{mV}$ and $V t=-45 \mathrm{mV}$.

\section{2) Hodgkin-Huxley neurons}

The Hodgkin-Huxley [18] model is widely considered as the benchmark standard for neural models. It is based upon experiments on the giant axon of the squid. Hodgkin and Huxley found three different types of ion current: sodium $\left(\mathrm{Na}^{+}\right)$, potassium $\left(\mathrm{K}^{+}\right)$, and a leak current that consists mainly of chloride $\left(\mathrm{Cl}^{-}\right)$ions. Different voltage-dependent ion channels control the flow of ions through the cell membrane. From their experiments, Hodgkin and Huxley formulated the following equation defining the time evolution of the model:

$$
\begin{gathered}
C \frac{d V}{d t}=g_{K} n^{4}\left(V-E_{K}\right)-g_{N a} m^{3} h\left(V-E_{N a}\right)-g_{L}\left(u-E_{L}\right) \\
\frac{d n}{d t}=\alpha_{n}(V)(1-n)-\beta_{n}(V) n \\
\frac{d m}{d t}=\alpha_{m}(V)(1-m)-\beta_{m}(V) m \\
\frac{d h}{d t}=\alpha_{h}(V)(1-h)-\beta_{h}(V) h
\end{gathered}
$$

$C$ is the capacitance and $n, m$ and $h$ describe the voltage dependence opening and closing dynamics of the ion channels. The maximum conductance of each channel are: $g_{k}=120$, $g_{N a}=36$ and $g_{L}=0.3$. The reversal potentials are set so that that $E_{k}=-12, E_{N a}=115$ and $E_{L}=10.6$. The rate functions for each channel are:

$$
\alpha_{n}(V)=\frac{(0.1-0.01 \mathrm{v})}{\exp (1.0-0.1 \mathrm{v})-1.0}
$$

$$
\begin{gathered}
\beta_{n}(V)=0.125 \exp \left(\frac{-v}{80.0}\right) \\
\alpha_{m}(V)=\frac{2.5-0.1 \mathrm{v}}{\exp (2.5-0.1 \mathrm{v})-1.0} \\
\beta_{m}(V)=4.0 \exp \left(\frac{-v}{18.0}\right) \\
\alpha_{h}(V)=0.07 \exp \left(\frac{-v}{20.0}\right) \\
\beta_{h}(V)=\frac{1.0}{\exp (3.0-0.1 \mathrm{v})+1.0}
\end{gathered}
$$

All work in this paper using the $\mathrm{HH}$ model adjusts the neuron resting potential from $0 \mathrm{mV}$ of the standard $\mathrm{HH}$ implementation to the more accepted value of $-65 \mathrm{mV}$ [29]

\section{B. Synaptic model}

The synaptic model for experiments using the QIF model simply multiplies the incoming spike by a synaptic weight, whereas the $\mathrm{HH}$ model uses synaptic reversal potentials to further scale incoming spikes. The latter model is as follows:

$$
s_{i j}(t)=w_{i j} t_{i}\left(\operatorname{Rev}{ }_{i j}-V_{j}\right)
$$

where $S_{i j}(t)$ is the synaptic input from neuron $i$ to neuron $j$ at time $t, t_{i}$ is the spike from neuron $i$ at time $t$, and $w_{i j}$ is the weight of the synapse connecting the two neurons. $\operatorname{Rev}_{i j}$ is the reversal potential of the synapse and $V_{j}$ is the voltage of the target neuron. The reversal potentials for the model are set to the same values in all experiments. For excitatory inputs the value is $0 \mathrm{mV}$, and for inhibitory inputs the value is $-70 \mathrm{mV}$. Not using a synaptic reversal model for the QIF model is equivalent to using a synaptic reversal model with reversal potentials set to $+\infty \mathrm{mV}$ for excitatory neurons and $-\infty \mathrm{mV}$ for inhibitory neurons.

\section{Synchronization metrics}

We only calculated synchrony for the excitatory neuron layer in an oscillatory cluster. The spikes of each neuron in a cluster were binned over time, and then a Gaussian smoothing filter was passed over the binned data to produce a continuous time varying signal. Following this, we performed a Hilbert transform on the mean-centered filtered signal in order to identify its phase. The synchrony between each pair of clusters was then calculated as follows:

$$
\text { sync }_{j k}=\frac{1}{t M a x} \sum\left(\text { mean }\left(\exp \left(h t_{j}(t) i\right), \exp \left(h t_{k}(t) i\right)\right)\right)
$$


where $h t_{j}(t)$ and $h t_{k}(t)$ are the Hilbert transforms at time $t$ of clusters $j$ and $k$ respectively. $i$ is the square root of -1 and $t$ Max is the length of time of the simulation. Pairwise synchrony between oscillator $j$ and all other $Q-1$ oscillators in the collection is calculated as:

$$
\operatorname{pair}_{j}=\frac{1}{Q-1} \sum \text { sync }_{j k}
$$

The total synchrony of all oscillators $\Phi$ in the collection is simply:

$$
\Phi=\frac{1}{Q} \sum \text { pair }_{j}
$$

\section{Hardware acceleration}

Each of our experiments required 16000 neurons and $36,256,000$ synapses, entailing an immense computational burden. To cope with this, we used the NeMo neural network simulator, which processes neurons concurrently on general purpose graphics processing units (GPUs) [30]. The NeMo software permits the addition of user plugins for neural models, which allowed us to implement both QIF and HH models for the NeMo simulator facilitating the work presented here.

\section{ACKNOWLEDGMENTS}

We would like to thank Andreas Fidjeland for his support on the NeMo hardware acceleration. This work is sponsored by EPSRC grant EP/F033516/1 and doctorial award to David Bhowmik.

\section{REFERENCES}

[1] Y. Nir, L. Fisch, R. Mukamel, H. Gelbard-Sagiv, A. Arieli, I. Fried, and R. Malach (2007). "Coupling between neuronal firing rate, gamma LFP, and BOLD $\mathrm{AMRI}$ is related to interneuronal correlations." Current biology $\square$ : CB, 17(15), 1275-85.

[2] M.D. Fox, A.Z. Snyder, J.L. Vincent, M. Corbetta, D.C. Van Essen, \& M.E. Raichle (2005). "The human brain is intrinsically organized into dynamic, anticorrelated functional networks." Proceedings of the National Academy of Sciences of the United States of America, 102(27): 9673-8.

[3] O. Jensen, J. Kaiser, and J. Lachaux (2007). "Human gamma-frequency oscillations associated with attention and memory." Trends in Neurosciences, 30(7), 317-324.

[4] W.H.R. Miltner, C. Braun, A. Matthias, H. Witte, and E. Taub (1999). "Coherence of gamma-band EEG activity as a basis for associative learning." Nature, 397(6718), 434-436.

[5] M. Siegel, M.R. Warden, and E.K. Miller (2009). "Phase-dependent neuronal coding of objects in short-term memory." Proceedings of the National Academy of Sciences of the United States of America, 106(50), 21341-6.

[6] J. Lisman (2005). "The theta/gamma discrete phase code occuring during the hippocampal phase precession may be a more general brain coding scheme." Hippocampus. 2005;15(7):913-22.

[7] E. Nyhus and T. Curran (2010). "Functional role of gamma and theta oscillations in episodic memory." Neuroscience and biobehavioral reviews. 2010;34(7):1023-35.
[8] P. Fries (2005 ). "A mechanism for cognitive dynamics: neuronal communication through neuronal coherence." Trends in cognitive sciences. 2005;9(10):474-80.

[9] M. Shanahan (2010). "Metastable chimera states in communitystructured oscillator networks." Chaos, 20(1), 013108.

[10] J. Acebrón, L. Bonilla, C. Pérez Vicente, F. Ritort, and R. Spigler (2005). "The Kuramoto model: A simple paradigm for synchronization phenomena." Reviews of Modern Physics, 77(1), 137-185.

[11] M. Breakspear, S. Heitmann, and A. Daffertshofer (2010). "Generative models of cortical oscillations: neurobiological implications of the kuramoto model." Frontiers in human neuroscience, 4(November), $190 .$.

[12] Y. Kuramoto (1984). Chemical Oscillations, Waves and Turbulence. Springer-Verlag, Berlin, 1984.

[13] S. Strogatz (2000). "From Kuramoto to Crawford: exploring the onset of synchronization in populations of coupled oscillators". Physica D: Nonlinear Phenomena, 143(1-4), 1-20.

[14] J. Cabral, E. Hugues, O. Sporns and G. Deco (2011). "Role of Local Network Oscillations in Resting-State Functional Connectivity." NeuroImage. 2011

[15] B.A. Santos, X.E. Barandiaran, and P. Husbands (2011). "Metastable dynamical regimes in an oscillatory network modulated by an agent ' $s$ sensorimotor loop." Proc. IEEE Symposium on Artificial Life 2011.

[16] A. Jadbabaie, N. Motee, and M. Barahona. (2004). "On the Stability of the Kuramoto Model of Coupled Nonlinear Oscillators." Systems Engineering, 1-8.

[17] P.E. Latham, B.J. Richmond, P.G. Nelson, and S. Nirenberg (2000). "Intrinsic dynamics in neuronal networks. i. theory." Journal of Neurophysiology 83(2), 808-827.

[18] A.L. Hodgkin and A.F. Huxley (1952). "A quantitative description of ion currents and its applications to conduction and excitation in nerve membranes." J. Physiol. (Lond.), 117:500-544.

[19] I. Vida., M. Bartos, and P. Jonas (2006). "Shunting inhibition improves robustness of gamma oscillations in hippocampal interneuron networks by homogenizing firing rates." Neuron, 49(1), 107-17.

[20] E. Papp, X. Leinekugel, D.A. Henze, J. Lee and G. Buzsáki (2001). "The apical shaft of CAl pyramidal cells is under GABAergic interneuronal control." Neuroscience 102(4).

[21] A. Hasenstaub, Y. Shu, and B. Haider (2005). "Inhibitory postsynaptic potentials carry synchronized frequency information in active cortical networks." Neuron. 2005;47(3):423-35.

[22] B.R. Alger, R.A. Nicoll (1979). "GABA-mediated biphasic inhibitory responses in hippocampus." Nature 281, 315 - 317.

[23] P. Andersen P, R. Dingledine, L. Gjerstad, I.A. Langmoen, A.M. Laursen (1980). "Two different responses of hippocampal pyramidal cells to application of gamma-amino butyric acid." J Phisiol 1980 Aug;305:279-96.

[24] H. Markram, J. Lübke, M. Frotscher, A. Roth, B. Sakmann (1997). "Physiology and anatomy of synaptic connections between thick tufted pyramidal neurones in the developing rat neocortex." The Journal of physiology. 1997;500 ( Pt 2):409-40.

[25] D. Hansel, G. Mato, and C. Meunier (1995). "Synchrony in excitatory neural networks." Neural computation. 1995;7(2):307-37.

[26] B. Ermentrout, B. (1996). "Type I membranes, phase resetting curves, and synchrony." Neural Computation 8(5), 979-1001.

[27] G.B. Ermentrout, and N. Kopell (1986). "Parabolic bursting in an excitable system coupled with a slow oscillation." SIAM Journal on Applied Mathematics 46(2), 233..

[28] C. Börgers, and N. Kopell (2005). "Effects of noisy drive on rhythms in networks of excitatory and inhibitory neurons." Neural Computation 17(3), 557-608.

[29] W. Gerstner and W. Kistler (2002) "Spiking neuron models." Cambridge University Press, 2002..

[30] A.K. Fidjeland, and M. Shanahan (2010). "Accelerated Simulation of Spiking Neural Networks Using GPUs.” Proceedings IJCNN 2010. 